\title{
The convergence of the coupled cluster approach for $\mathrm{MgO}$
}

\author{
Charles W. Bauschlicher, Jr.* \\ NASA Ames Research Center, Moffett Field, CA 94035
}

\begin{abstract}
The $X^{1} \Sigma^{+}$state of $\mathrm{MgO}$ shows significant differences between the CCSD(T) and CCSDT results and even some significant differences between the CCSDT and CCSDTQ results. The CCSDT $(\mathrm{Q})$ results for this state do not agree well with the CCSDTQ results. Unlike the $X$ state, the $a^{3} \Pi$ state, which is well described by a single configuration, shows much less dependence on level of correlation treatment. Despite the slow convergence of the valence treatment of the $X$ state, the effect of core correlation converges rapidly with level of correlation treatment.
\end{abstract}

Keywords: CCSD(T), CCSDT, CCSDTQ, core correlation

* Mail Stop 230-3, Thermal Protection Materials Branch, Charles.W.Bauschlicher@nasa.gov 


\section{INTRODUCTION}

We recently studied ${ }^{1}$ the spectra of $\mathrm{MgO}$ using a dynamical weighted stateaveraged complete active space self-consistent-field/internally contracted multireference configuration interaction (DW-SA-CASSCF/IC-MRCI) approach ${ }^{2,3}$. To make the study of 25 electronic states practical, we only correlated the valence electrons. As part of this study, we considered effect of core correlation on some of the properties $\left(r_{e}, \omega_{e}, T_{e}\right.$, and $\left.D_{e}\right)$ of the two lowest states, namely, the $X^{1} \Sigma^{+}$and $a^{3} \Pi$ states, using the coupled cluster singles and doubles with a perturbative estimate for the triples, i.e. $\operatorname{CCSD}(\mathrm{T})^{4,5}$ approach. At the valence level, the $\operatorname{CCSD}(\mathrm{T})$ and IC-MRCI $r_{e}$ and $\omega_{e}$ results for the $a^{3} \Pi$ state were in very good agreement, while the $\operatorname{CCSD}(\mathrm{T})$ and IC-MRCI results for the $X^{1} \Sigma^{+}$state did not agree with experiment as well as for the $a$ state. This is consistent with the multireference character of the $X^{1} \Sigma^{+}$state, and suggests that one must go beyond the $\operatorname{CCSD}(\mathrm{T})$ approach to obtain more accurate results for the $X$ state. We should note however, that while the $\operatorname{CCSD}(\mathrm{T})$ and ICMRCI $r_{e}, \omega_{e}$, and $T_{e}$ results differed, the effect of core correlation computed at the $\operatorname{CCSD}(\mathrm{T})$ level was similar to the difference between the IC-MRCI and experiment. Thus suggesting that the $\operatorname{CCSD}(\mathrm{T})$ approach might give a good estimate for the core correlation, even though the valence results were disappointing.

Consistent with our findings on $\mathrm{MgO}$, Martin and co-workers have found ${ }^{6-8}$ that to

obtain extremely accurate thermochemistry, it is often necessary to use higher levels of coupled cluster theory than CCSD(T). For example, they considered coupled cluster theory including triples and quadruples, CCSDT and CCSDTQ, and a version where the quadruples were included perturbatively, $\operatorname{CCSDT}(\mathrm{Q})$. We should note that in their study, they also found $\mathrm{MgO} X^{1} \Sigma^{+}$to be one of the more difficult systems to describe.

In this work, we return to the $X^{1} \Sigma^{+}$and the $a^{3} \Pi$ states of $\mathrm{MgO}$ and study the effect of going beyond $\operatorname{CCSD}(\mathrm{T})$ on the properties of these two states, one of which is well described by a single configuration and the other, which is not. We consider treatments that include valence and core+valence correlation. 


\section{METHODS}

The $X^{1} \Sigma^{+}$state is a closed-shell system and a spin-restricted Hartree-Fock (RHF) reference is used in all calculations. The $a^{3} \Pi$ state is an open-shell system and both spin-restricted (RHF) and spin-unrestricted HF (UHF) based approaches are used. Several levels of coupled cluster calculations are performed. The simplest approach is the $\operatorname{CCSD}(\mathrm{T})^{4,5}$. For the $a^{3} \Pi$ state, the RHF based approach is the $\operatorname{RCCSD}(\mathrm{T})$ described by Knowles et al. ${ }^{9}$, while the UHF based approach is the usual UCCSD(T) method. The next level is the CCSDT approach ${ }^{10}$ that includes the triples iteratively. The CCSDT $(\mathrm{Q})$ includes a perturbative estimate of the quadruples ${ }^{11}$. Our final level, CCSDTQ, includes the quadruples iteratively ${ }^{12}$. We correlate the valence electrons, i.e. the $\mathrm{Mg} 3 s$ and oxygen $2 s$ and $2 p$ electrons in our valence treatment and all electrons except the $\mathrm{Mg} 1 s$ electrons in our core+valence treatment.

The correlation consistent basis sets developed by Peterson and Dunning and their co-workers ${ }^{13-16}$ are used in all calculations. The valence correlation treatment uses augmented-correlation-consistent polarized valence triple zeta (aug-cc-pVTZ), quadruple zeta (aug-cc-pVQZ) and quintuple zeta (aug-cc-pV5Z) basis sets. To study the effect of core correlation, the correlation-consistent polarized core-valence (ccpCV) basis sets are used; the same basis sets are used for valence and core+valence calculations so the core correlation effects can be computed without any complications from the use of different basis sets. Test calculations show that the diffuse functions on $\mathrm{Mg}$ have virtually no effect on the results, which is consistent with the $\mathrm{Mg}^{2+} \mathrm{O}^{2-}$ or $\mathrm{Mg}^{+} \mathrm{O}^{-}$character of the states. Therefore, the augmented functions are only added to the oxygen atom in these calculations. We use the double zeta, triple zeta, quadruple zeta, and quintuple zeta versions of these basis sets. That is, we use the aug-cc-pCVDZ, aug-cc-pCVTZ, aug-cc-pCVQZ, and aug-cc-pCV5Z sets for oxygen and the cc-pCVDZ, cc-pCVTZ, cc-pCVQZ, and cc-pCV5Z sets for Mg. We denote these basis sets as (aug)-cc-pCVnZ, where the aug is in parentheses to indicate that the diffuse functions are only added to the oxygen atom.

We test the effect of relativity using the Douglas-Kroll approximation ${ }^{17,18}$. The $\operatorname{CCSD}(\mathrm{T})$ ( $X$ state) and $\mathrm{RCCSD}(\mathrm{T})$ (a state) approaches are used in conjunction 
with aug-cc-pVTZ-DK basis sets. As we show below, the effect of relativity is very small on the properties considered in this work, and therefore most of the calculations ignore relativistic effects.

The geometries were optimized and harmonic frequencies were computed using a parabolic fit in $r$ and $1 / r$. The points used in the fit are $r_{e}$ and $r_{e} \pm 0.01$ or $r_{e} \pm 0.02 \AA$. Tests show that the bond lengths should be accurate to about $0.001 \AA$ and the $T_{e}$ values to about $1 \mathrm{~cm}^{-1}$. While the $\omega_{e}$ values computed using the $r$ and $1 / r$ agree to better than $1 \mathrm{~cm}^{-1}$, varying the spacing between the points can lead to differences of a few $\mathrm{cm}^{-1}$. Therefore in the tables, variations in the $\omega_{e}$ values should be given much less weight than changes in $r_{e}$ and $T_{e}$.

The $X^{1} \Sigma^{+} \operatorname{CCSD}(\mathrm{T})$ calculations and $a^{3} \Pi \operatorname{RCCSD}(\mathrm{T})$ calculations are performed using MOLPRO ${ }^{19}$. The $a^{3} \Pi$ RCCSDT calculations are performed using MOLPRO interfaced with the MRCC program ${ }^{20}$. All of the other calculations are performed using the MRCC program, except for the $X^{1} \Sigma^{+} \operatorname{CCSD}(\mathrm{T})$ and $a^{3} \Pi \operatorname{UCCSD}(\mathrm{T})$ calculations in the (aug)-cc-pCV5Z basis set which are performed using Gaussian16 ${ }^{21}$.

\section{RESULTS AND DISCUSSION}

The results of the valence level treatments are summarized in Table I. The top two entries compare $\operatorname{CCSD}(\mathrm{T})$ calculations with and without relativistic corrections. The $r_{e}$ values are unchanged and the $T_{e}$ and $\omega_{e}$ values are hardly changed. Clearly relativistic effects make a small difference on the properties considered in this work and are therefore ignored in the rest of the calculations.

The $r_{e}$ and $\omega_{e}$ values for the $a^{3} \Pi$ state do not strongly depend on the level of correlation treatment used. For the aug-cc-pVTZ basis set, the $r_{e}$ value varies by $0.002 \AA$ with correlation method, while the $\omega_{e}$ value varies by about $3 \mathrm{~cm}^{-1}$. Improving the basis set, reduces the $r_{e}$ value and increases the $\omega_{e}$ value; the difference between the UCCSDT values in aug-cc-pVTZ and aug-cc-pV5Z basis sets are $0.011 \AA$ and $10.8 \mathrm{~cm}^{-1}$, respectively. That is, the change with basis set is five times larger than that found for changing the level of correlation treatment. For the $X^{1} \Sigma^{+}$state, the variation with level of correlation treatment is about the same size as the vari- 
ation with basis set. That is, the $X^{1} \Sigma^{+}$state is much more sensitive to the level of correlation treatment, which is consistent with the fact that the $X$ state is not as well described by a single configuration as the $a$ state. Given the fact that the two states are not equally well described by a single reference, it is not surprising that the $T_{e}$ value is sensitive to the correlation treatment.

For the $X$ state the CCSDT and $\operatorname{CCSD}(\mathrm{T})$ results shows some significant differences; the CCSDT has a longer bond length and smaller $\omega_{e}$ value than does the $\operatorname{CCSD}(\mathrm{T})$. Also note the change in $T_{e}$ between these two methods. While difference in the $r_{e}$ and $\omega_{e}$ values between the CCSDT and CCSDTQ are smaller than found between the $\operatorname{CCSD}(\mathrm{T})$ and $\mathrm{CCSDT}$, the $T_{e}$ value show a surprisingly large effect; see the results for the aug-cc-pVTZ basis set in Table I. Given that the $X$ state is not well described by a single configuration, it is not unexpected that the perturbative and iterative description of triples differ. However, it somewhat disappointing that the CCSDT(Q) $T_{e}$ values do not agree well with that obtained at the CCSDTQ level. We further note that CCSDT(Q) suggest the quadruples increases $r_{e}$ by $0.002 \AA$ and decreases $\omega_{e}$ by $10.7 \mathrm{~cm}^{-1}$, while the CCSDTQ shows an increase of $0.001 \AA r_{e}$ and an increase of $2.3 \mathrm{~cm}^{-1}$ in $\omega_{e}$.

Our highest level calculation is the CCSDT/aug-cc-pV5Z approach. For the $a^{3} \Pi$ state this level yields $r_{e}$ and $\omega_{e}$ values are in excellent agreement with our previous results $^{1}$ using the IC-MRCI approach in conjunction with the same basis set, see Table I. The $X$ state shows somewhat larger differences between the two methods, but CCSDT is a very large improvement over the $\operatorname{CCSD}(\mathrm{T})$ approach. The ICMRCI $T_{e}$ value is actually closer to the $\operatorname{CCSD}(\mathrm{T})$ result then the $\operatorname{CCSDT}$ value. The CCSDTQ results in the TZ basis set suggests that the $\operatorname{CCSD}(\mathrm{T})$ is benefitting from a cancelation of errors as the inclusion of quadruples would increase the CCSDT value by more than $500 \mathrm{~cm}^{-1}$. The CCSDT value can be improved by extrapolating ${ }^{22}$ to the complete basis set (CBS) limit and adding on an estimate for the effect of quadruples using the aug-cc-pVTZ basis set, namely

$$
\text { CCSDT/CBS + CCSDTQ/aug-cc-pVTZ - CCSDT/aug-cc-pVTZ, }
$$

which is denoted "CBS+Q(TZ)" in the table. This estimate is compared with experiment $^{23}$ in Table I. Note that we also give the CCSDT/aug-cc-pV5Z values to show 
the size of the CBS extrapolation. Our best valence estimates differ from experiment. Since we showed relativistic effects are small, the difference with experiment probably arises from core-correlation, and we therefore next consider this effect.

The results including core correlation are shown in Table II, along with the valence results obtained using the same basis set. The agreement of the valence treatments using the aug-cc-pVnZ and (aug)-cc-pCVnZ basis set is very good. While the tight functions added to the aug-cc-pVnZ basis set to form the (aug)-cc-pCVnZ sets are added for inner shell correlation, they have have some effect on the valence results, in this regard, we note that (aug)-cc-pCVTZ results fall between those obtained using the (aug)-cc-pVTZ and (aug)-cc-pVQZ sets.

The results obtained in the bottom of the table include core correlation. The trends with basis set improvement and level of correlation treatment follow those discussed above for the valence treatment. Of more interest is the effect of the core correlation, and these results are summarize in Table III, which are obtained by taking the difference between the valence and core+valence results obtained using the (aug)cc-pCVnZ basis sets; that is, as differences between the results in the top and bottom of Table II. While the effect of core correlation increases with basis set improvement, for a given basis set, the effect of core correlation are much less dependent on the level of correlation than are the values themselves. For example the difference between the $\operatorname{CCSD}(\mathrm{T})$ and CCSDTQ $T_{e}$ values in the (aug)-cc-pCVDZ basis set is only about $17 \mathrm{~cm}^{-1}$. Since the CCSD $(\mathrm{T})$ and CCSDT $T_{e}$ values approximately double between the (aug)-cc-pCVDZ and (aug)-cc-pCVQZ basis sets, the effect of the quadruples is probably underestimated using the (aug)-cc-pCVDZ basis set, but it is safe to conclude that the effect of quadruples is small. This is encouraging as it is then possible to correct high level valence treatment for core effects using lower levels of theory.

Our best valence level results show some significant differences with experiment as did our previous IC-MRCI results. This suggest that most of the difference with experiment is due to core correlation. However, the additional electrons to be correlated, means that the inclusion of triples and quadruples must be performed in smaller basis sets than for the valence treatment. This means that our best estimate will have to 
a composite of several treatments. We take our CCSDT/(aug)-cc-pCVQZ results as our best directly computed values for the effect of core correlation. This value should be corrected for basis set incompleteness and higher level correlation. The basis set incompleteness can be estimated as the difference between the $\operatorname{CCSD}(\mathrm{T}) / \operatorname{RCCSD}(\mathrm{T})$ or CCSD(T)/UCCSD(T) levels using the (aug)-cc-pCVQZ and (aug)-cc-pCV5Z basis sets. Since the CCSDT/(aug)-cc-pCVQZ core calculations are very expensive, we also try using the CCSDT/(aug)-cc-pCVTZ results with a basis set correction computed using the CCSD(T) values for the (aug)-cc-pCVTZ and (aug)-cc-pCV5Z basis sets. Higher excitations can be estimated using the CCSDTQ and CCSDT results performed with the (aug)-cc-pCVDZ set; this suggests that quadruples increase $T_{e}$ by $15 \mathrm{~cm}^{-1}$ and has virtually no effect on the other properties. Since the $\operatorname{CCSD}(\mathrm{T}) /($ aug)-cc-pCVDZ yields about half the core effect as CCSD(T)/(aug)-ccpCV5Z, we double the $T_{e}$ value computed using the smaller basis set, and conclude that quadruples would increase $T_{e}$ by about $30 \mathrm{~cm}^{-1}$.

We add our estimates for the core correlation effects to our best estimate for the valence results to obtain our best estimates; these results are summarized in Table IV. For comparison, our valence estimates are included in the top of the table and experiment at the bottom. We first note that using the $\operatorname{RCCSD}(\mathrm{T})$ or $\mathrm{UCCSD}(\mathrm{T})$ approach to estimate the basis set incompleteness yields very similar results. In addition, using the smaller CCSDT/(aug)-cc-pCVTZ result corrected with CCSD(T)(5Z-TZ) estimate for core correlation basis set incompleteness, instead of the CCSDT/(aug)-cc-pCVQZ + CCSD(T)(5Z-QZ), makes a very small difference. This is very encouraging as the CCSDT/(aug)-cc-pCVTZ is significantly cheaper than the CCSDT/(aug)-cc-pCVQZ calculation. It is also interesting to note that just using the CCSD(T)/(aug)-cc-pCV5Z approach, without any CCSDT calculations gives a good estimate for the core correlation. That is, while the $\operatorname{CCSD}(\mathrm{T})$ approach is not sufficient for the valence description of the $X$ state, it does give a reasonable estimate for the core effects. All of the results including a estimate for the core correlation are in reasonable agreement with experiment.

All of the results using CCSDT/CBS+Q for the valence result and the CCSDT based results for the core effect have about a $30 \mathrm{~cm}^{-1}$ error in the $T_{e}$ value. This 
increases a bit if the CCSDT $/ 5 \mathrm{Z}+\mathrm{Q}$ is used for the valence or if only the $\operatorname{CCSD}(\mathrm{T})$ approach is used from the core contribution, but all are a big improvement over the valence result. The $a$ state $r_{e}$ value agrees with experiment if the CBS based result is used and slightly long if the $5 \mathrm{Z}$ based result is used. The $5 \mathrm{Z}$ based $X$ state $r_{e}$ value is slightly too long while the CBS yields a $r_{e}$ value that is too short, but both values significantly better than the valence results. Because of the uncertainty in the $\omega_{e}$ values, it is harder to make any definitive statements other than that all of the methods give reasonable results, and the values for the $X$ state are an improvement on the valence results.

\section{CONCLUSIONS}

The CCSD(T), CCSDT, and CCSDTQ results for the $a^{3} \Pi$ state are in good agreement, as expected for a state well described by a single reference configuration. For the $X^{1} \Sigma^{+}$state, which is not as well described by a single reference configuration, the CCSDT and $\operatorname{CCSD}(\mathrm{T})$ results differ. For this state, there is also a significant difference between the CCSDT and CCSDTQ. The CCSDT(Q) results differ with those obtained using the CCSDTQ. Unlike the valence treatment, it appears that there is a cancellation of errors such that the effect of core correlation is much easier

to describe. Even the $\operatorname{CCSD}(\mathrm{T})$ approach gives a reasonable result for effect of core correlation. This is encouraging for composite methods of computing properties as adding core correlation can dramatically increase the cost of the calculation.

\section{ACKNOWLEDGMENTS}

This work was funded by NASA.

1 C.W. Bauschlicher, D.W. Schwenke, Chem. Phys. Letts. 683 (2017) 62-67.

2 M.P. Deskevich, D.J. Nesbitt, H.-J. Werner, J. Chem. Phys. 120 (2004) 7281. 
3 H.-J. Werner, P.J. Knowles, J. Chem. Phys. 89 (1988) 5803; P.J. Knowles, H.-J. Werner, Chem. Phys. Lett. 145 (1988) 514.

4 R. J. Bartlett, Annu. Rev. Phys. Chem. 32 (1981) 359.

5 K. Raghavachari, G.W. Trucks, J.A. Pople, M.A. Head-Gordon, Chem. Phys. Lett. 157 (1989) 479.

6 J.M.L. Martin, G. de Oliveira, J. Chem. Phys. 111 (1999) 1843-1856.

7 A.D. Boese, M. Oren, O. Atasoylu, J.M.L. Martin, M. Kállay, J. Gauss, J. Chem. Phys. 120 (2004) 4129-4141.

8 A. Karton, E. Rabinovich, J.M.L. Martin, B. Ruscic, J. Chem. Phys. 125 (2006) 144108.

9 P.J. Knowles, C. Hampel, H.-J. Werner, J. Chem. Phys. 99 (1993) 5219 (see also erratum, J. Chem. Phys., 112 (2000) 3106)

10 J. Noga, R.J. Bartlett, J. Chem. Phys. 86 (1987) 7041.

11 Y.J. Bomble, J.F. Stanton, M. Kállay, J. Gauss, J. Chem. Phys. 123 (2005) 054101.

12 S.A. Kucharski , R.J. Bartlett, J. Chem. Phys. 97 (1992) 4282.

13 T. H. Dunning, J. Chem. Phys. 90 (1989)1007-1023.

14 R.A. Kendall, T.H. Dunning, R.J. Harrison, J. Chem. Phys. 96 (1992) 6796-6806.

15 D.E. Woon, T. H. Dunning, J. Chem. Phys. 103 (1995) 4572.

16 B.P. Prascher, D.E. Woon, K.A. Peterson, T.H. Dunning, A.K. Wilson, Theor. Chem. Acc. 128 ( 2011) 69-82.

17 M. Douglas, N.M. Kroll, Ann. Phys. N.Y. 82 (1974) 89.

18 B.A. Hess, Phys. Rev. A 33 (1986) 3742.

19 MOLPRO, version 2010.1, a package of ab initio programs, H.-J. Werner, P. J. Knowles, F. R. Manby, M. Schütz, P. Celani, G. Knizia, T. Korona, R. Lindh, A. Mitrushenkov, G. Rauhut, T. B. Adler, R. D. Amos, A. Bernhardsson, A. Berning, D. L. Cooper, M. J. O. Deegan, A. J. Dobbyn, F. Eckert, E. Goll, C. Hampel, A. Hesselmann, G. Hetzer, T. Hrenar, G. Jansen, C. Köppl, Y. Liu, A. W. Lloyd, R. A. Mata, A. J. May, S. J. McNicholas, W. Meyer, M. E. Mura, A. Nicklass, P. Palmieri, K. Pflüger, R. Pitzer, M. Reiher, T. Shiozaki, H. Stoll, A. J. Stone, R. Tarroni, T. Thorsteinsson, M. Wang, A. Wolf, see http://www.molpro.net.

20 MRCC, a quantum chemical program suite written by M. Kállay, Z. Rolik, J. Csontos, P. 
Nagy, G. Samu, D. Mester, J. Csóka, I. Ladjánszki, L. Szegedy, B. Ladóczki, K. Petrov, M. Farkas, and B. Hégely. See also Z. Rolik, L. Szegedy, I. Ladjánszki, B. Ladóczki, and M. Kállay, J. Chem. Phys. 139, 094105 (2013), as well as: www.mrcc.hu.

21 M.J. Frisch, et al., Gaussian16, Revision A.03, Gaussian, Inc., Wallingford CT, 2016.

22 T. Helgaker, W. Klopper, H. Koch, J. Noga, J. Chem. Phys. 106 (1997) 9639-9646.

23 K.P. Huber, G. Herzberg, 1979 "Molecular Spectra and Molecular Structure: IV. Constants of Diatomic Molecules," Van Nostrand Reinhold Company. 
TABLE I: Summary of spectroscopic constants for the valence treatment of correlation.

\begin{tabular}{|c|c|c|c|c|c|c|}
\hline \multicolumn{3}{|c|}{$X^{1} \Sigma^{+}$} & \multicolumn{4}{|c|}{$a^{3} \Pi$} \\
\hline Method & $r_{e}(\AA)$ & $\omega_{e}\left(\mathrm{~cm}^{-1}\right)$ & Method & $r_{e}(\AA)$ & $\omega_{e}\left(\mathrm{~cm}^{-1}\right)$ & $T_{e}\left(\mathrm{~cm}^{-1}\right)$ \\
\hline \multicolumn{7}{|c|}{ aug-cc-pVTZ-DK } \\
\hline $\operatorname{CCSD}(\mathrm{T})-\mathrm{DK}$ & 1.766 & 769.1 & $\operatorname{RCCSD}(\mathrm{T})-\mathrm{DK}$ & 1.894 & 632.2 & 1687.6 \\
\hline $\operatorname{CCSD}(\mathrm{T})$ & 1.766 & 771.5 & $\operatorname{RCCSD}(T)$ & 1.894 & 633.7 & 1679.9 \\
\hline \multicolumn{7}{|c|}{ aug-cc-pVTZ } \\
\hline \multirow[t]{2}{*}{$\operatorname{CCSD}(\mathrm{T})$} & 1.766 & 770.4 & $\operatorname{RCCSD}(\mathrm{T})$ & 1.895 & 632.9 & 1677.6 \\
\hline & & & $\operatorname{UCCSD}(\mathrm{T})$ & 1.895 & 634.9 & 1608.0 \\
\hline \multirow[t]{2}{*}{ CCSDT } & 1.777 & 753.8 & RCCSDT & 1.896 & 633.3 & 1340.2 \\
\hline & & & UCCSDT & 1.896 & 632.7 & 1321.2 \\
\hline $\operatorname{CCSDT}(\mathrm{Q})$ & 1.779 & 743.1 & UCCSDT(Q) & 1.896 & 631.5 & 2182.0 \\
\hline CCSDTQ & 1.778 & 756.1 & UCCSDTQ & 1.896 & 631.9 & 1860.5 \\
\hline \multicolumn{7}{|c|}{ aug-cc-pVQZ } \\
\hline $\operatorname{CCSD}(\mathrm{T})$ & 1.759 & 788.5 & $\operatorname{RCCSD}(\mathrm{T})$ & 1.888 & 642.8 & 1864.5 \\
\hline \multirow[t]{2}{*}{ CCSDT } & 1.770 & 763.1 & RCCSDT & 1.889 & 641.0 & 1487.1 \\
\hline & & & UCCSDT & 1.889 & 640.4 & 1466.2 \\
\hline $\operatorname{CCSDT}(\mathrm{Q})$ & 1.772 & 752.7 & UCCSDT(Q) & 1.889 & 639.2 & 2335.0 \\
\hline \multicolumn{7}{|c|}{ aug-cc-pV5Z } \\
\hline $\operatorname{CCSD}(\mathrm{T})$ & 1.755 & 796.9 & $\operatorname{RCCSD}(\mathrm{T})$ & 1.884 & 645.8 & 1999.5 \\
\hline CCSDT & 1.765 & 765.5 & UCCSDT & 1.885 & 643.5 & 1571.2 \\
\hline $\operatorname{CCSDT}(\mathrm{Q})$ & 1.768 & 756.9 & UCCSDT(Q) & 1.885 & 642.3 & 2439.7 \\
\hline IC-MRCI $^{1}$ & 1.769 & 768.6 & IC-MRCI $^{1}$ & 1.884 & 643.7 & 1932.8 \\
\hline \multicolumn{7}{|c|}{ Best Estimates } \\
\hline $5 \mathrm{Z}+\mathrm{Q}(\mathrm{TZ})$ & 1.766 & 767.7 & & 1.885 & 642.7 & 2110.5 \\
\hline $\mathrm{CBS}+\mathrm{Q}(\mathrm{TZ})$ & 1.762 & 770.2 & & 1.881 & 646.0 & 2220.7 \\
\hline Expt & 1.749 & 785.0 & & 1.868 & 650.2 & 2621.0 \\
\hline
\end{tabular}


TABLE II: Summary of spectroscopic constants for valence and core treatment of correlation.

\begin{tabular}{|c|c|c|c|c|c|c|}
\hline \multicolumn{3}{|c|}{$X^{1} \Sigma^{+}$} & \multicolumn{3}{|c|}{$a^{3} \Pi$} & \multirow[b]{2}{*}{$T_{e}\left(\mathrm{~cm}^{-1}\right)$} \\
\hline \multicolumn{6}{|c|}{ Valence correlation } & \\
\hline \multicolumn{7}{|c|}{ (aug)-cc-pCVDZ } \\
\hline $\operatorname{CCSD}(\mathrm{T})$ & 1.782 & 751.1 & $\operatorname{RCCSD}(\mathrm{T})$ & 1.908 & 624.9 & 943.3 \\
\hline CCSDT & 1.793 & 740.8 & UCCSDT & 1.910 & 621.1 & 759.1 \\
\hline CCSDTQ & 1.794 & 743.1 & UCCSDTQ & 1.910 & 620.3 & 1240.6 \\
\hline \multicolumn{7}{|c|}{ (aug)-cc-pCVTZ } \\
\hline \multirow[t]{2}{*}{$\operatorname{CCSD}(\mathrm{T})$} & 1.760 & 783.6 & $\operatorname{RCCSD}(\mathrm{T})$ & 1.890 & 636.5 & 1795.3 \\
\hline & & & $\mathrm{UCCSD}(\mathrm{T})$ & 1.890 & 635.7 & 1722.8 \\
\hline \multirow[t]{2}{*}{ CCSDT } & 1.771 & 756.4 & RCCSDT & 1.891 & 633.7 & 1382.4 \\
\hline & & & UCCSDT & 1.891 & 633.5 & 1402.4 \\
\hline $\operatorname{CCSDT}(\mathrm{Q})$ & 1.773 & 746.1 & UCCSDT(Q) & 1.891 & 632.3 & 2261.0 \\
\hline \multicolumn{7}{|c|}{ (aug)-cc-pCVQZ } \\
\hline \multirow[t]{2}{*}{$\operatorname{CCSD}(\mathrm{T})$} & 1.755 & 797.3 & $\operatorname{RCCSD}(\mathrm{T})$ & 1.884 & 644.9 & 1973.2 \\
\hline & & & $\operatorname{UCCSD}(\mathrm{T})$ & 1.884 & 644.2 & 1898.5 \\
\hline CCSDT & 1.766 & 765.3 & UCCSDT & 1.885 & 643.2 & 1548.1 \\
\hline \multicolumn{7}{|c|}{ (aug)-cc-pCV5Z } \\
\hline \multirow[t]{2}{*}{$\operatorname{CCSD}(\mathrm{T})$} & 1.754 & 798.5 & $\operatorname{RCCSD}(\mathrm{T})$ & 1.883 & 650.9 & 2027.4 \\
\hline & & & $\operatorname{UCCSD}(\mathrm{T})$ & 1.883 & 645.1 & 1953.0 \\
\hline \multicolumn{7}{|c|}{ Core+Valence correlation } \\
\hline \multicolumn{7}{|c|}{ (aug)-cc-pCVDZ } \\
\hline $\operatorname{CCSD}(\mathrm{T})$ & 1.774 & 757.3 & $\operatorname{RCCSD}(\mathrm{T})$ & 1.903 & 617.5 & 1125.2 \\
\hline CCSDT & 1.786 & 744.1 & UCCSDT & 1.905 & 619.1 & 942.3 \\
\hline CCSDTQ & 1.786 & 746.0 & UCCSDTQ & 1.906 & 618.2 & 1439.2 \\
\hline \multicolumn{7}{|c|}{ (aug)-cc-pCVTZ } \\
\hline \multirow[t]{2}{*}{$\operatorname{CCSD}(\mathrm{T})$} & 1.748 & 796.2 & $\operatorname{RCCSD}(\mathrm{T})$ & 1.880 & 640.2 & 2088.5 \\
\hline & & & $\mathrm{UCCSD}(\mathrm{T})$ & 1.881 & 639.3 & 2031.4 \\
\hline \multirow[t]{2}{*}{ CCSDT } & 1.759 & 765.2 & RCCSDT & 1.882 & 637.1 & 1694.9 \\
\hline & & & UCCSDT & 1.882 & 637.1 & 1694.9 \\
\hline $\operatorname{CCSDT}(\mathrm{Q})$ & 1.760 & 757.0 & UCCSDT(Q) & 1.882 & 635.7 & 2590.2 \\
\hline \multicolumn{7}{|c|}{ (aug)-cc-pCVQZ } \\
\hline \multirow[t]{2}{*}{$\operatorname{CCSD}(\mathrm{T})$} & 1.741 & 811.8 & $\operatorname{RCCSD}(\mathrm{T})$ & 1.872 & 648.8 & 2298.7 \\
\hline & & & $\mathrm{UCCSD}(\mathrm{T})$ & 1.873 & 649.7 & 2239.6 \\
\hline CCSDT & 1.751 & 777.7 & UCCSDT & 1.873 & 648.7 & 1873.1 \\
\hline \multicolumn{7}{|c|}{ (aug)-cc-pCV5Z } \\
\hline $\operatorname{CCSD}(\mathrm{T})$ & 1.739 & 814.5 & $\operatorname{RCCSD}(\mathrm{T})$ & 1.870 & 652.6 & 2368.3 \\
\hline & & & $\operatorname{UCCSD}(\mathrm{T})$ & 1.870 & 651.6 & 2309.2 \\
\hline
\end{tabular}


TABLE III: The effect of core correlation.

\begin{tabular}{|c|c|c|c|c|c|}
\hline & $X^{1} \Sigma^{+}$ & & $a^{3} \Pi$ & & \\
\hline Method & $r_{e}(\AA)$ & $\omega_{e}\left(\mathrm{~cm}^{-1}\right) \quad$ Method & $r_{e}(\AA)$ & $\omega_{e}\left(\mathrm{~cm}^{-1}\right)$ & $T_{e}\left(\mathrm{~cm}^{-1}\right)$ \\
\hline & & (aug)-cc-pCVD & & & \\
\hline $\operatorname{CCSD}(\mathrm{T})$ & -0.008 & $6.2 \operatorname{RCCSD}(\mathrm{T})$ & -0.005 & -7.4 & 181.9 \\
\hline CCSDT & -0.008 & $3.4 \mathrm{UCCSDT}$ & -0.005 & -2.0 & 183.2 \\
\hline CCSDTQ & -0.008 & 2.9 UCCSDTQ & -0.004 & -2.2 & 198.6 \\
\hline & & (aug)-cc-pCVT & & & \\
\hline $\operatorname{CCSD}(\mathrm{T})$ & -0.012 & 12.6 $\operatorname{RCCSD}(\mathrm{T})$ & -0.009 & 3.7 & 293.3 \\
\hline & & $\operatorname{UCCSD}(\mathrm{T})$ & -0.009 & 3.6 & 308.5 \\
\hline CCSDT & -0.012 & 8.8 RCCSDT & -0.009 & 3.4 & 312.5 \\
\hline & & UCCSDT & -0.009 & 3.6 & 292.5 \\
\hline $\operatorname{CCSDT}(\mathrm{Q})$ & -0.013 & $10.9 \operatorname{UCCSDT}(\mathrm{Q})$ & -0.009 & 3.5 & 329.2 \\
\hline & & (aug)-cc-pCVQ & & & \\
\hline $\operatorname{CCSD}(\mathrm{T})$ & -0.014 & $14.4 \operatorname{RCCSD}(\mathrm{T})$ & -0.012 & 3.9 & 325.5 \\
\hline & & $\mathrm{UCCSD}(\mathrm{T})$ & -0.011 & 5.5 & 341.0 \\
\hline CCSDT & -0.015 & $12.5 \mathrm{UCCSDT}$ & -0.011 & 5.5 & 325.1 \\
\hline & & (aug)-cc-pCV5 & & & \\
\hline $\operatorname{CCSD}(\mathrm{T})$ & -0.015 & 16.0 $\operatorname{RCCSD}(\mathrm{T})$ & -0.013 & 1.6 & 340.8 \\
\hline & & $\operatorname{UCCSD}(\mathrm{T})$ & -0.013 & 6.5 & 356.1 \\
\hline
\end{tabular}


TABLE IV: Best estimates for the spectroscopic constants. $r_{e}$ is in $\AA$ and $\omega_{e}$ and $T_{e}$ are in $\mathrm{cm}^{-1}$.

$$
\begin{aligned}
& \text { Method } \\
& \text { Valence }^{a} \quad \text { Core }^{b} \\
& 5 \mathrm{Z}+\mathrm{Q}(\mathrm{TZ}) \\
& \frac{X^{1} \Sigma^{+}}{r_{e} \quad \omega_{e}} \frac{a^{3} \Pi}{r_{e} \quad \omega_{e} \quad T_{e}} \\
& 1.766767 .71 .885642 .72110 .5 \\
& \mathrm{CBS}+\mathrm{Q}(\mathrm{TZ}) \\
& 1.762770 .21 .881646 .02220 .7 \\
& \text { 5Z+Q(TZ) QZ+RCCSD(T)(5Z-QZ)+Q(DZ)*2 } 1.751781 .81 .872646 .02480 .9 \\
& \text { 5Z+Q(TZ) QZ+UCCSD(T)(5Z-QZ)+Q(DZ)*2 } 1.751781 .81 .872649 .22480 .7 \\
& \text { 5Z+Q(TZ) TZ+RCCSD(T)(5Z-TZ)+Q(DZ)*2 } 1.751779 .91 .872644 .32480 .7 \\
& \text { 5Z+Q(TZ) TZ+UCCSD(T)(5Z-TZ)+Q(DZ)*2 } 1.751779 .91 .872649 .22480 .6 \\
& \text { CBS+Q(TZ) QZ+RCCSD(T)(5Z-QZ)+Q(DZ)*2 } 1.746784 .31 .868649 .32591 .1 \\
& \text { CBS+Q(TZ) QZ+UCCSD(T)(5Z-QZ)+Q(DZ)*2 } 1.746784 .31 .868652 .52590 .9 \\
& \text { CBS+Q(TZ) TZ+RCCSD(T)(5Z-TZ)+Q(DZ)*2 } 1.746782 .31 .868647 .62590 .8 \\
& \text { CBS+Q(TZ) TZ+UCCSD(T)(5Z-TZ)+Q(DZ)*2 } 1.746782 .31 .868652 .52590 .8 \\
& 5 \mathrm{Z}+\mathrm{Q}(\mathrm{TZ}) \quad \mathrm{RCCSD}(\mathrm{T})(5 \mathrm{Z})+\mathrm{Q}(\mathrm{DZ})^{*} 2 \quad 1.751783 .81 .872644 .42451 .4 \\
& 5 \mathrm{Z}+\mathrm{Q}(\mathrm{TZ}) \quad \mathrm{UCCSD}(\mathrm{T})(5 \mathrm{Z})+\mathrm{Q}(\mathrm{DZ})^{*} 2 \quad 1.751783 .81 .872649 .32466 .7 \\
& \begin{array}{ll}
\mathrm{CBS}+\mathrm{Q}(\mathrm{TZ}) \mathrm{RCCSD}(\mathrm{T})(5 \mathrm{Z})+\mathrm{Q}(\mathrm{DZ})^{*} 2 & 1.746786 .21 .868647 .62561 .6
\end{array} \\
& \text { CBS+Q(TZ) UCCSD(T)(5Z)+Q(DZ)*2 } \quad 1.746786 .21 .868652 .52576 .8 \\
& \text { Expt } \\
& 1.749785 .01 .868650 .22621 .0
\end{aligned}
$$

$a$ "5Z" denotes the CCSDT/aug-cc-pV5Z results and $+\mathrm{Q}(\mathrm{TZ})$ denotes the effect for quadruples compute as the difference between the CCSDTQ and CCSDT in the aug-cc-pVTZ basis set. "CBS" indicates the CCSDT is extrapolated complete basis set limit.

$b$ "QZ" denotes the core effect computed using the CCSDT/(aug)-cc-pCVQZ approach, while "TZ" indicates the analogous result from (aug)-cc-pCVTZ basis set. The (R or U) CCSD(T)(X,Y) indicates that the $\operatorname{CCSD}(\mathrm{T})$ and $(\mathrm{R}$ or $\mathrm{U}) \operatorname{CCSD}(\mathrm{T})$ approach using the $\mathrm{X}$ and $\mathrm{Y}$ basis sets where used to estimate the basis incompleteness for the core correlation. "(R or U) CCSD(T)(5Z)" indicates the effect of core correlation is computed using the (R or $\mathrm{U}) \operatorname{CCSD}(\mathrm{T}) /($ aug $)$-cc-pCV5Z basis set. "Q(DZ)" indicates the effect of quadruples in the (aug)-cc-pCVDZ basis set. 